\title{
RASSF7 negatively regulates pro-apoptotic JNK signaling by inhibiting the activity of phosphorylated-MKK7
}

\author{
S Takahashi ${ }^{1,2,3}$, A Ebihara ${ }^{1,3}$, H Kajiho ${ }^{1}$, K Kontani $^{1}$, H Nishina ${ }^{2}$ and T Katada*,1
}

Members of the Ras-association domain family (RASSF) of proteins influence apoptosis and cell cycling but little is known about the mechanisms. Here, we show that RASSF7 interacts with N-Ras and mitogen-activated protein kinase kinase 7 (MKK7) to negatively regulate c-Jun N-terminal kinase (JNK) signaling. Stress-induced JNK activation and apoptosis were markedly enhanced in cells depleted of RASSF7 or N-Ras by RNAi knockdown. An interaction with RASSF7 promoted the phosphorylated state of MKK7 but inhibited this kinase's ability to activate JNK. RASSF7 required its RA domain for both interaction with GTP-bound N-Ras and the anti-apoptotic response to stress stimuli. Following prolonged stress, however, RASSF7's antiapoptotic effect was eliminated because of degradation of RASSF7 protein via the ubiquitin-proteasome pathway. Our results indicate that RASSF7 acts in concert with N-Ras to constitute a stress-sensitive temporary mechanism of apoptotic regulation. With initial stress, RASSF7/N-Ras promotes cell survival by inhibiting the MKK7/JNK pathway. However, with prolonged stress, RASSF7 protein undergoes degradation that allows cell death signaling to proceed. Our findings may account for the association of elevated RASSF7 with tumorigenesis.

Cell Death and Differentiation (2011) 18, 645-655; doi:10.1038/cdd.2010.137; published online 19 November 2010

Members of the Ras-association domain family (RASSF) share a conserved motif called the RalGDS/AF6-type Ras association (RA) domain. ${ }^{1-3}$ The vertebrate RASSF comprises the classical RASSF members, RASSF1-6, and the more recently identified N-terminal (NT) RASSF members, RASSF7-10. ${ }^{1-3}$ Epigenetic silencing of most of RASSF genes has been observed at high frequencies in various tumor types. ${ }^{1,2}$ In contrast, upregulation of RASSF7 has been noted in pancreatic, endometrial and ovarian cancers, ${ }^{4-8}$ although no direct evidence exists indicating that RASSF7 promotes tumorigenesis.

RASSF7 was originally identified as HRAS cluster 1 (HRC1), located within a cluster of genes situated about 32-kb upstream of HRAS1 on human chromosome 11p15. In Xenopus, knockdown of the RASSF7 homolog prevented cells from completing mitosis and caused a striking loss of tissue architecture in the neural tube. ${ }^{9}$ However, the physiological functions of RASSF7 in mammals are unknown, and the signaling pathways in which RASSF7 participates remain a mystery.

The c-Jun N-terminal kinase (JNK) enzymes function primarily in stress-activated signaling and are important as both positive and negative modulators of apoptosis, depending on the cellular context. ${ }^{10,11}$ In response to cell stresses, such as heat shock, ultraviolet (UV) irradiation, hyperosmolarity and ischemia/reperfusion injury, MAP kinase kinase kinases (MAPKKKs) are activated. These enzymes then activate MAP kinase kinase 4 (MKK4; also known as SEK1) and/or MKK7, which in turn activate JNKs. ${ }^{12,13}$ The extent and specificity of JNK activation serve as important signals for promoting the stabilization and transcriptional activity of JNK substrates, which then mediate cell survival and/or apoptosis. Although phosphatases capable of dephosphorylating JNK cascade substrates have been deemed the primary negative regulators of JNK signaling, ${ }^{14,15}$ several other proteins inhibit mitogen-activated kinase kinases (MAPKKs) signaling by interacting physically with various cascade components. ${ }^{16-18}$ Thus, the precise molecular mechanism(s) that modulate JNK activation have yet to be completely elucidated.

That RASSF proteins contribute to the control of JNK signaling has been known for a decade but the mechanisms remain unclear. ${ }^{19}$ We previously reported that the classical RASSF member RASSF1C is required for the prolonged JNK activation that promotes UV-induced cell death. ${ }^{20}$ In this study, we show that RASSF7, an NT RASSF, negatively regulates JNK signaling by inhibiting the catalytic activity of MKK7.

\section{Results}

UV-induced cellular apoptosis is enhanced by RASSF7 knockdown. To identify the role of RASSF7 in the regulation of intracellular signaling, we first performed knockdown

\footnotetext{
${ }^{1}$ Department of Physiological Chemistry, Graduate School of Pharmaceutical Sciences, University of Tokyo, Tokyo, Japan and ${ }^{2}$ Department of Developmental and Regenerative Biology, Medical Research Institute, Tokyo Medical and Dental University, Tokyo, Japan

*Corresponding author: T Katada, Department of Physiological Chemistry, University of Tokyo, Graduate School of Pharmaceutical Sciences, 7-3-1 Hongo, Bunkyo-ku, Tokyo 113-0033, Japan. Tel: + 8135841 4753; Fax: + 8135841 4751; E-mail: katada@ mol.f.u-tokyo.ac.jp

${ }^{3}$ These authors contributed equally to this work.

Keywords: RASSF; JNK signaling; stress-induced apoptosis

Abbreviations: RASSF, Ras-association domain family; JNK, c-Jun N-terminal kinase; MAPK, mitogen-activated kinase; MAPKK, mitogen-activated kinase kinase; MAPKKK, mitogen-activated kinase kinase kinase; UV, ultraviolet

Received 31.3.10; revised 13.9.10; accepted 13.9.10; Edited by C Borner; published online 19.11 .10
} 
experiments in HeLa cells. The successful knockdown of RASSF7 by the RNA silencing method (siRNA) was confirmed by immunoblotting using a monoclonal antibody $(\mathrm{Ab})$ against recombinant full-length RASSF7 (Figure 1a). To test whether RASSF7 regulated cell survival or apoptosis, we analyzed UV-induced caspase activation in control and RASSF7-knockdown cells. Apoptosis was monitored by the appearance of the cleaved form of caspase-3, as well as cleaved caspase-9 and cleaved poly-ADP-ribosyl polymerase (PARP), which are substrates of caspase-3. In the absence of UV exposure, the cleaved forms of these molecules were barely detectable in both control and RASSF7-knockdown cells (Figure 1b, lanes 1 versus 3). However, UV irradiation induced the marked accumulation of the cleaved forms in RASSF7-knockdown cells but not in control cells (Figure 1b, lanes 2 versus 4). Immunostaining to detect cleaved caspase-3 confirmed that UV-induced apoptosis was enhanced fivefold by RASSF7 knockdown (Figures 1c and d). This increase in apoptosis associated with RASSF7 knockdown correlated both with increased UV intensity (Figure 1e) and elapsed time from UV exposure (Figure 1f). In addition to their marked accumulation of cleaved caspase-3, UV-irradiated RASSF7-knockdown cells exhibited morphological characters of apoptosis, including nuclear condensation and membrane blebbing (data not shown). These findings indicate that RASSF7 normally acts to inhibit UV-induced apoptosis in HeLa cells.

UV-induced JNK activation leading to apoptosis is specifically enhanced by RASSF7-knockdown. It has been reported that UV-induced apoptosis involves the activation of two mitogen-activated kinases (MAPKs), namely JNK and p38. ${ }^{21}$ We therefore investigated whether UV-induced MAPK activation was affected by RASSF7 knockdown. JNK phosphorylation (p-JNK; activated) was rapidly elevated in both control and RASSF7-knockdown cells after UV irradiation (Figure 2a). However, this elevation was sustained only in the absence of RASSF7, with levels of $\mathrm{p}$-JNK in control cells rapidly returning to baseline levels by $2 \mathrm{~h}$. In contrast, RASSF7 knockdown had no effect on UV-induced elevation of phosphorylated p38 (p-p38; Figure $2 b$ ). This specific enhancement of UV-induced JNK activation in RASSF7-knockdown cells was confirmed using an in vitro kinase activity assay. Control and RASSF7-knockdown cells were transfected with FLAG-JNK1 and exposed to UV. FLAG-JNK1 was then isolated from these cells and incubated in vitro with glutathione S-transferase (GST)-C-Jun, which is a substrate of JNK. C-Jun phosphorylation was a

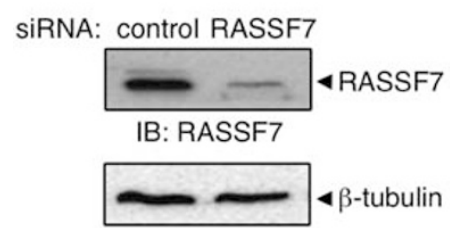

IB: $\beta$-tubulin
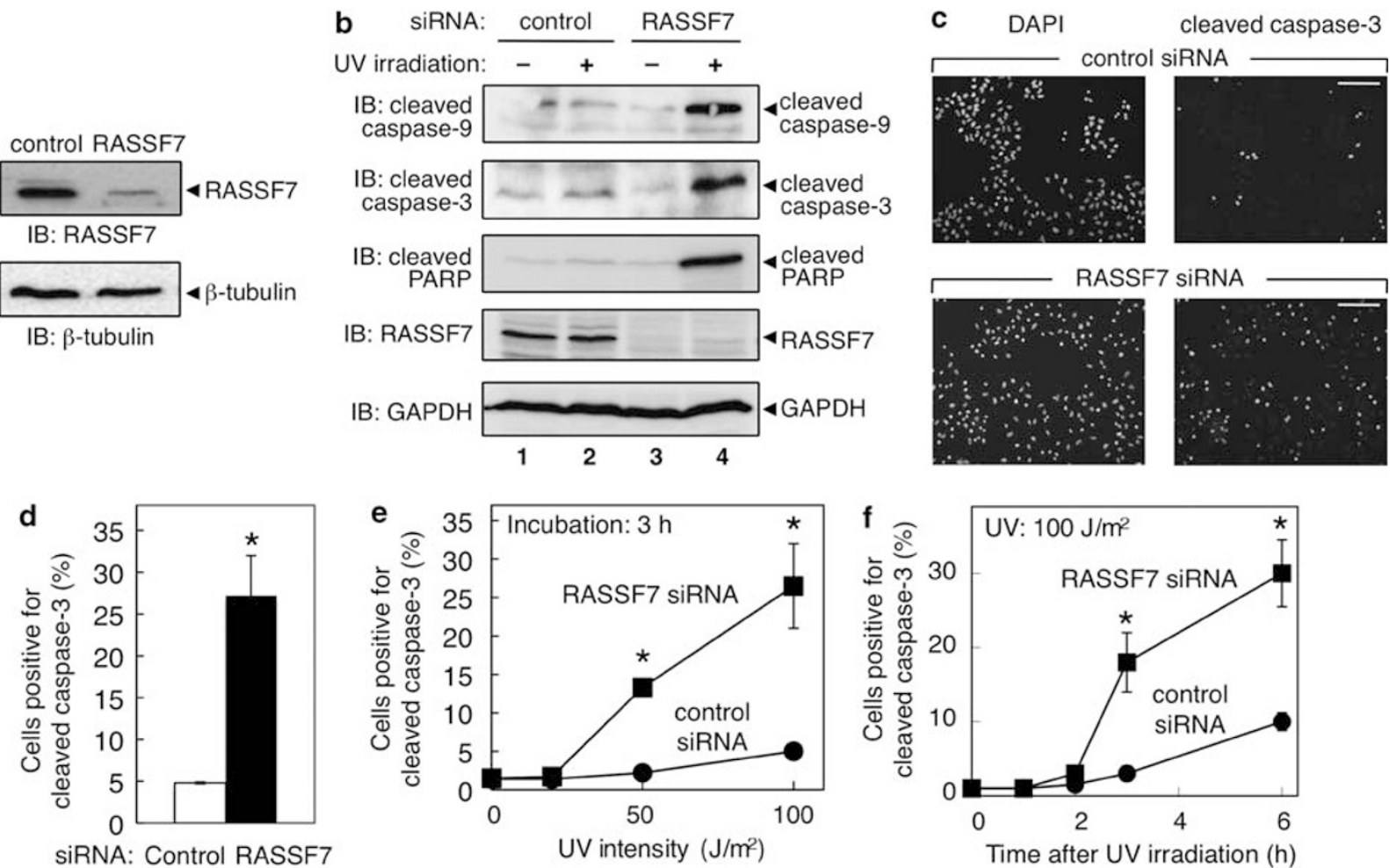

Figure 1 RASSF7-knockdown cells are highly sensitive to UV-induced apoptosis. (a) Validation of RASSF7 siRNA. HeLa cells were treated with control or RASSF7 siRNA. Lysates were immunoblotted to detect RASSF7 and $\beta$-tubulin (loading control). (b) Increased cleavage of apoptotic mediators. HeLa cells treated with control or RASSF7 siRNA were exposed to UV $\left(100 \mathrm{~J} / \mathrm{m}^{2}\right)$ and cultured for $6 \mathrm{~h}$. Lysates were immunoblotted to detect the indicated proteins. GADPH, loading control. (c and d) Quantitation of increased caspase-3 cleavage. HeLa cells treated with control or RASSF7 siRNA were exposed to UV (100 J/m²) and cultured for $3 \mathrm{~h}$. (c) Cells were fixed and stained with DAPI (left) or immunostained with an Ab recognizing cleaved caspase-3 plus Alexa-Fluor 488 anti-rabbit lgG (right). Scale bar: $100 \mu \mathrm{M}$. (d) The percentage of total DAPI-stained cells in $c$ that were also positive for Alexa-488 was determined from three fields of 100-200 cells in each of two independent experiments. Values shown are the mean \pm S.D. ${ }^{*} P<0.05$, compared with control siRNA. (e and f) Correlation with UV exposure. HeLa cells treated with control or RASSF7 siRNA were exposed to the indicated intensities of UV for $3 \mathrm{~h}(\mathbf{e})$, or to $100 \mathrm{~J} / \mathrm{m}^{2} \mathrm{UV}$ for the indicated times (f). The number of cells positive for cleaved caspase-3 was determined as for $\mathbf{c}$ and $\mathbf{d}$. Values shown are the mean \pm S.D. ${ }^{*} P<0.05$, compared with control siRNA 

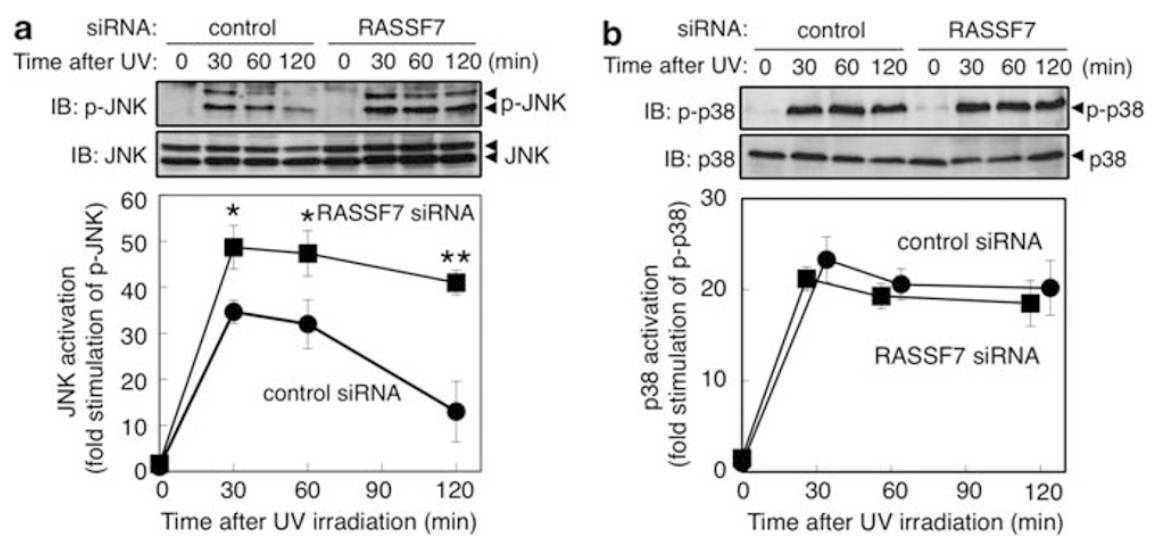

C
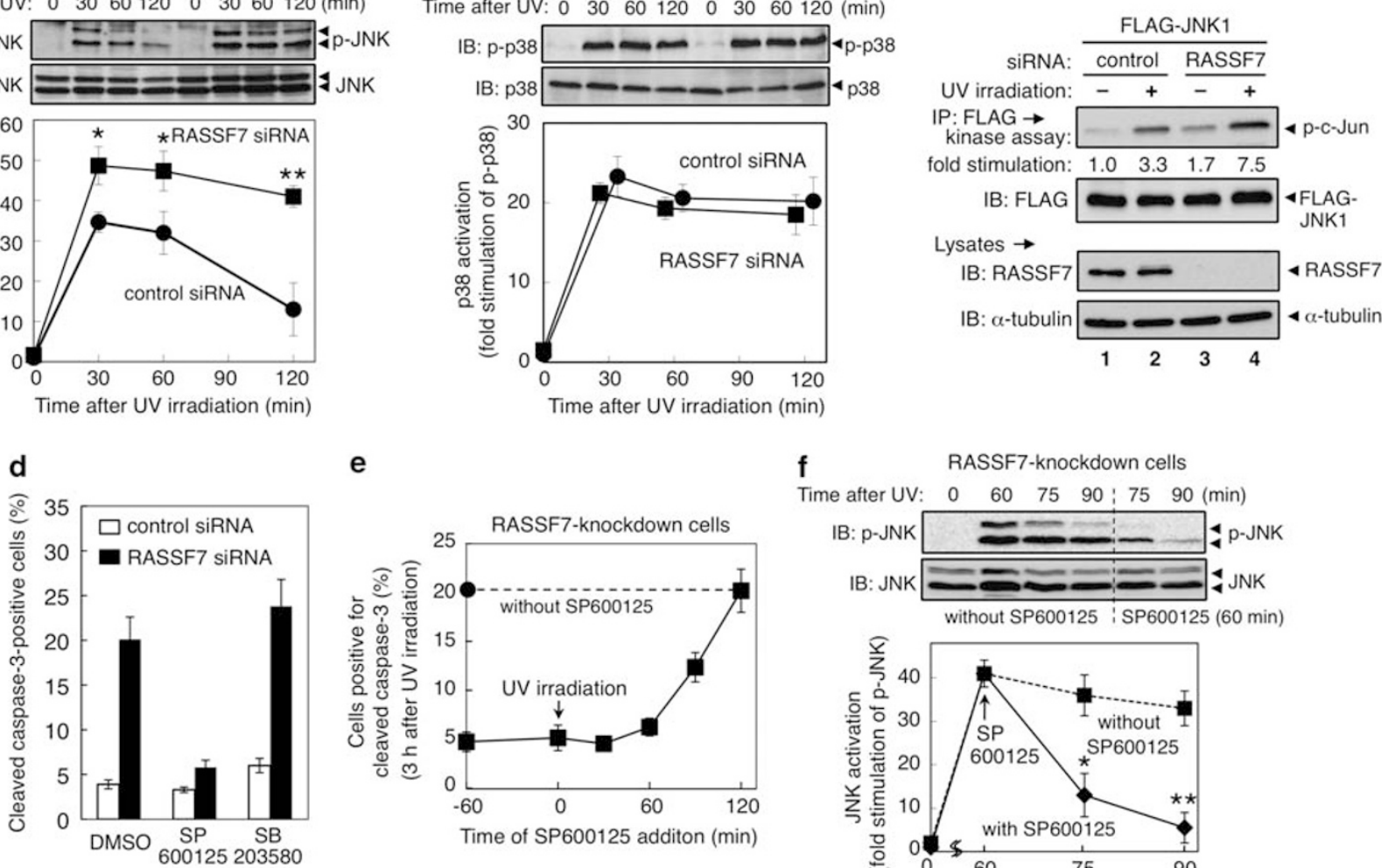

e
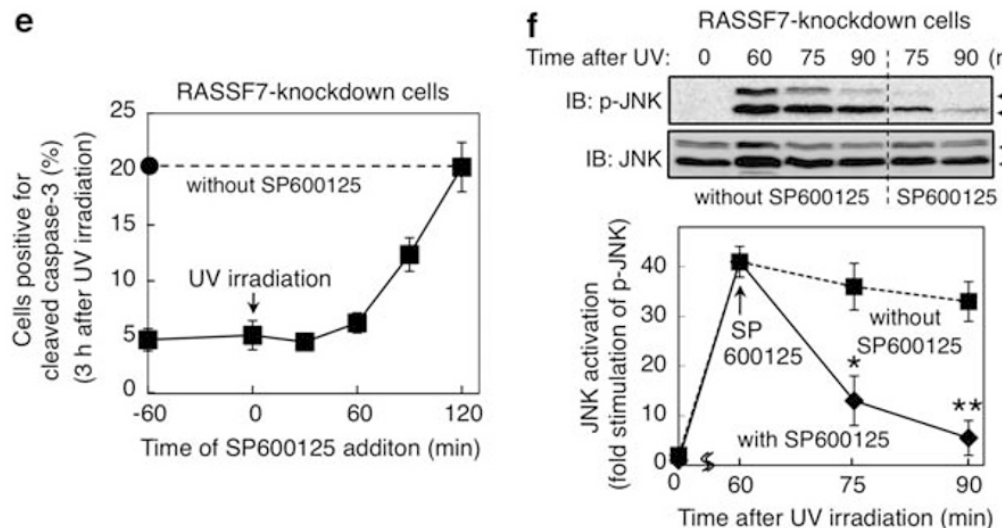

Figure 2 UV-induced JNK activation leading to apoptosis is specifically enhanced by RASSF7 knockdown. (a and $\mathbf{b}$ ) Increased p-JNK but unaltered p-p38. HeLa cells treated with control or RASSF7 siRNA were exposed to UV $\left(100 \mathrm{~J} / \mathrm{m}^{2}\right)$ and cultured for the indicated times. Lysates were immunoblotted (top) to detect total and phosphorylated JNK (p-JNK; a), and total and phosphorylated p38 (p-p38; b). Results were quantitated by densitometry from blots and expressed as mean fold stimulation (phosphorylated/total) \pm S.D. over levels in control cultures not exposed to UV. ${ }^{*} P<0.05$ and ${ }^{\star *} P<0.01$, compared with control siRNA. (c) Increased c-Jun phosphorylation. HeLa cells treated with control or RASSF7 siRNA for $24 \mathrm{~h}$ were transfected with FLAG-JNK1 for $24 \mathrm{~h}$. Cells were exposed to UV $\left(100 \mathrm{~J} / \mathrm{m}^{2}\right)$ and cultured for $1 \mathrm{~h}$. Immunoprecipitated JNK1 using anti-FLAG beads were subjected to an in vitro kinase assay using GST-C-Jun as the substrate. The original lysates, the immunoprecipitates and the assay products were immunoblotted to detect the indicated proteins. $\alpha$-tubulin, loading control. Fold stimulation, densitometric evaluation of the relative increase in lanes 2-4 over lane 1. (d) Specificity for JNK. HeLa cells treated with control or RASSF7 siRNA were pretreated with DMSO (control) or $20 \mu \mathrm{M} \mathrm{SP600125} \mathrm{(JNK} \mathrm{inhibitor)} \mathrm{or}$ SB203580 (p38 inhibitor) for $1 \mathrm{~h}$. Cells were then exposed to UV $\left(100 \mathrm{~J} / \mathrm{m}^{2}\right)$ and cultured for $3 \mathrm{~h}$ with inhibitors. Cells positive for cleaved caspase-3 were evaluated as for Figures $1 \mathrm{c}$ and d. (e) Late phase effect on cleaved caspase-3. HeLa cells treated with RASSF7 siRNA were exposed to UV $\left(100 \mathrm{~J} / \mathrm{m}^{2}\right)$ at time 0 and incubated for $3 \mathrm{~h}$. During the incubation, SP600125 $(20 \mu \mathrm{M})$ was also added at times indicated. Numbers of cells positive for cleaved caspase-3 were determined as for $\mathbf{d}$. (f) Late phase effect on p-JNK. HeLa cells treated with RASSF7 siRNA were exposed to UV $\left(100 \mathrm{~J} / \mathrm{m}^{2}\right)$ and cultured for the indicated times. SP600125 $(20 \mu \mathrm{M})$ was added at $60 \mathrm{~min}$ post-UV. JNK activation was quantitated as for $\mathbf{a} .{ }^{*} P<0.05$ and ${ }^{* *} P<0.01$, compared with without SP600125

substantially increased in the absence of RASSF7 (Figure 2c, lanes 2 versus 4), indicating that the activation of JNK, rather than p38, is specifically enhanced in RASSF7knockdown cells.

We next used MAPK-specific inhibitors to investigate whether the apoptosis-promoting property of RASSF7 knockdown was truly because of enhanced JNK activation. Preincubation with the JNK inhibitor SP600125 abolished the stimulation of UV-induced apoptosis observed in RASSF7knockdown cells, but addition of the p38 inhibitor SB203580 did not (Figure 2d). The effectiveness and specificity of SP600125 inhibition were confirmed by immunoblot analyses to detect p-JNK and p-p38 (Supplementary Figure S1). When SP600125 was added at $60 \mathrm{~min}$ after UV irradiation, the accumulation of cleaved caspase- 3 assayed at $3 \mathrm{~h}$ post-UV was not observed in RASSF7-knockdown cells (Figure 2e). Concomitantly, the UV-induced elevation in JNK activation seen in RASSF7-knockdown cells was still effectively and rapidly diminished (Figure 2f). We confirmed that these RASSF7-knockdown effects were reproducible by using another RASSF7-targeted SiRNA, whose sequence is totally different (Supplementary Figure S2). Furthermore, we confirmed that the UV-induced apoptosis was also enhanced by RASSF7 knockdown in HaCaT keratinocytes, which have been generated from the human skin, directly exposed to UV from the sun. (Supplementary Figure S3). These results strongly suggest that the sustained activation of JNK is possible in the absence of RASSF7 that drives the apoptosis of UV-irradiated cells.

The phosphorylated state of MKK7 is promoted by physical interaction with RASSF7. Stress-induced activation of JNK requires its phosphorylation by the upstream MAPKKs MKK4 and MKK7..$^{22-25}$ Several regulatory proteins 
inhibit MAPK activity by interacting with upstream components of the JNK pathways. ${ }^{16-18}$ To investigate whether RASSF7 associated with JNK or its upstream kinases, we transiently co-transfected 293T cells with control vector or FLAG-tagged RASSF7, plus myc-tagged JNK1, -MKK4 or -MKK7. We then subjected these cells to immunoprecipitation using anti-FLAG beads. As shown in Figure 3a, MKK7 specifically immunoprecipitated with RASSF7 (lane 6) but MKK4 (lane 4) and JNK (lane 2) did not. The direct interaction between RASSF7 and MKK7 was confirmed using in vitro-binding experiments with their recombinant proteins. For the analysis, GST-fused RASSF7 and MKK7 were purified from E. coli, and their GST tags were cleaved by precision protease and applied to GST pull-down assay. The purified MKK7 was capable of interacting with GST-RASSF7 (Figure 3b, lane 2), but not with GST alone (lane 1). In the same way, the purified RASSF7 could interact with GST-MKK7 (Figure 3c). Furthermore, the interaction between RASSF7 and MKK7 was confirmed with the endogenous proteins in $293 \mathrm{~T}$ cells. When the endogenous
RASSF7 was precipitated with RASSF7-specific Ab (Figure 3d), MKK7 was co-precipitated (lane 2). Interestingly, this interaction was enhanced with UV treatment (lane 4). To clarify whether phosphorylation of MKK7 is important for interacting with RASSF7, we constructed MKK7 proteins with mutations in the phosphorylation sites (S271, T275 and S277) in the activation loop motif. We also devised a constitutively active mutant (ST/DE) mimicking MKK7's phosphorylated form, as well as constitutively inactive (ST/AA) and nonphosphorylatable (STS/AAA) mutants of MKK7. RASSF7 preferentially interacted with the phospho-mimic MKK7 mutant (Figure 3e, lane 2) rather than wild-type (WT) MKK7 (lane 1) or the dephosphorylated MKK7 mutants (lanes 3 and 4). We then examined whether RASSF7 can affect phosphorylation state of MKK7. To describe this, we separated phosphorylated and nonphosphorylated MKK7 more clearly by dephosphorylation assay. As shown in Figure 3f, a visible shift in the size of the MKK7 band was evident with the expression of RASSF7 (lane 3 ), and that was reduced on previous incubation of

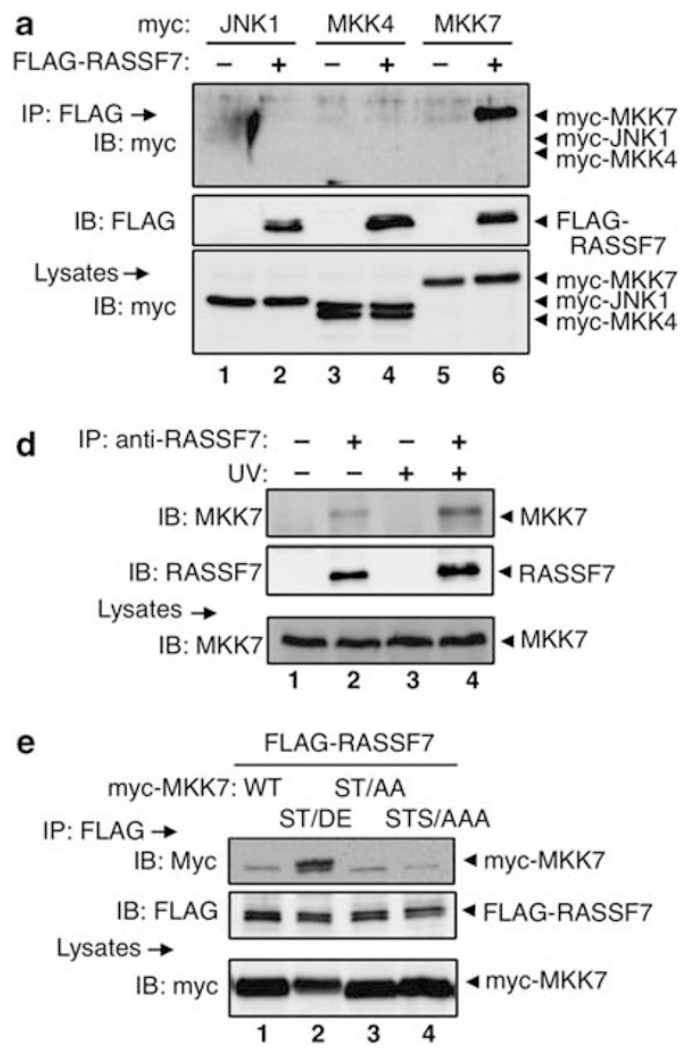

b

C
Pull-down: GST $\rightarrow$ purified MKK 7 GST: mock RASSF7
IB: MKK7 $\square-M K K 7$ Input $\rightarrow$

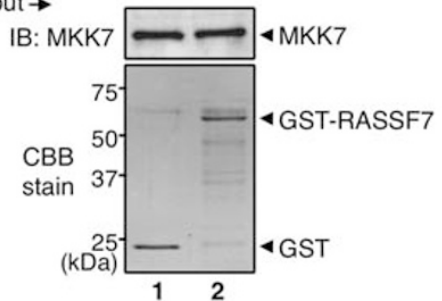
Pull-down: GST $\rightarrow$ purified RASSF7 GST: mock MKK7
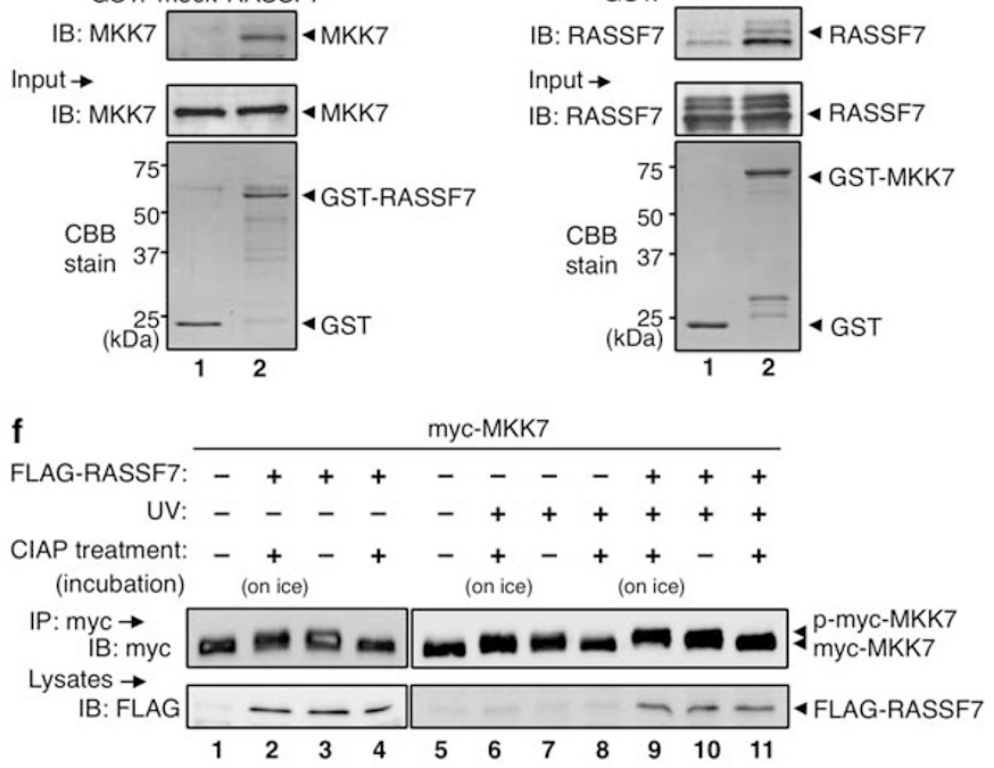

Figure 3 The phosphorylated state of MKK7 is promoted by physical interaction with RASSF7. (a) Specific binding to MKK7. 293T cells were co-transfected with FLAG-mock or FLAG-RASSF7, plus myc-JNK1, -MKK4 or -MKK7. Lysates and precipitated proteins with anti-FLAG beads were immunoblotted to detect the indicated proteins. (b and $\mathbf{c}$ ) In vitro binding of RASSF7 with MKK7. GST-fused RASSF7, MKK7 and GST alone were purified from E. coli using glutathione beads, analyzed by SDSPAGE, and stained with Coomassie Brilliant Blue (lower panel). The beads were combined with the GST-cleaved recombinant of the other, prepared by precision protease (middle panel). Precipitated proteins with the beads were detected using a specific Ab. The results of GST-cleaved MKK7 with GST-fused RASSF7 (b) and GST-cleaved RASSF7 with GST-fused MKK7 (c) were shown. (d) Endogenous binding of MKK7 with RASSF7. 293T cells were exposed to UV (100 J/m²) and cultured for $1 \mathrm{~h}$. Lysates and precipitated proteins with anti-rat IgG or anti-RASSF7 bound beads were immunoblotted to detect the indicated proteins. (e) Phosphorylation includes RASSF7 binding to MKK7. 293T cells were transiently co-transfected with FLAG-RASSF7 plus myc-MKK7 enzymes that were wild-type (WT), constitutively active (ST/DE), constitutively inactive (ST/AA) or non-phosphorylatable (STT/AAA), and cultured for $48 \mathrm{~h}$. Lysates and precipitated proteins with anti-FLAG beads were immunoblotted to detect the indicated proteins. (f) Preference for phospho-MKK7. 293T cells co-transfected with myc-MKK7 plus FLAG-mock or FLAG-RASSF7 were exposed to UV (100 J/m²) and cultured for $1 \mathrm{~h}$. Lysates and precipitated proteins with anti-myc Ab were subjected to CIAP treatment at $37^{\circ} \mathrm{C}$ or on ice before immunoblotting to detect the indicated proteins 
immunoprecipitated beads with a phosphatase (calf intestine alkaline phosphatase $(\mathrm{CIAP})$ ) at $37^{\circ} \mathrm{C}$ (lane 4) but not when the beads were incubated with CIAP on ice (lane 2), indicating that the band-shift was due to phosphorylation. A similar shift in the MKK7 band was observed in response to UV irradiation in the absence of RASSF7 (lane 7), whereas the shift was further enhanced by co-transfection with RASSF7 (lane 10). Both of these band-shifts were again reduced by CIAP treatment (lanes 8 and 11). Thus, RASSF7 overexpression and/or UV irradiation favor the phosphorylated state of MKK7, either by stimulating its phosphorylation or by inhibiting its dephosphorylation. These results show that RASSF7 physically interacts with phosphorylated MKK7, and suggest that this interaction may protect phosphorylated MKK7 from its dephosphorylation.

RASSF7 inhibits the ability of stress-stimulated MKK7 to phosphorylate JNK. To determine whether the direct interaction between RASSF7 and MKK7 could inhibit JNK activation, we analyzed the kinase activities of MKKs under cells exhibiting either overexpression or knockdown of RASSF7. First, we transfected HeLa cells with FLAG-MKK7 or FLAG-MKK4 and exposed them to UV irradiation. The MKK4 and MKK7 proteins were isolated using FLAG beads and subjected to in vitro kinase assays using GST-JNK1 as the substrate. The ectopic expression of RASSF7 completely abolished UV-stimulated MKK7 activity (Figure 4a, lanes 6 versus 8) without altering UV-stimulated MKK4 activity (lanes 2 versus 4), although the stress-induced MKK7 band-shift observed on RASSF7 expression was maintained (see Figure 3f). These findings again suggest that MKK7 is the specific target of RASSF7 in the JNK signaling cascade. We then confirmed the role of endogenous RASSF7 in regulating MKK7 using siRNA. In control siRNA-transfected cells, MKK7 activity was enhanced by UV irradiation (Figure $4 \mathrm{~b}$, lane 6). In RASSF7 siRNA-transfected cells, the elevated MKK7 activity that was already present under resting conditions (lane 7) was further enhanced by UV irradiation (lane 8). In contrast, MKK4 activity was not affected by RASSF7 knockdown (Figure 4b, lanes 2 versus 4). Moreover, the addition of partially purified RASSF7 directly to a reaction mixture inhibited UV-stimulated MKK7 activity (Figure 4c, lanes 6 versus 8), without significantly affecting MKK4 activity (lane 2 versus 4). Constitutively active MKK7 can phosphorylate JNK in the absence of activation of upstream MAPKKKs. ${ }^{26,27}$ We found that phosphorylation of JNK mediated by constitutively active MKK7 was inhibited by RASSF7 co-expression (Figure 4d, lanes 1 versus 2). Taken together, these results indicate that RASSF7 can directly inhibit the catalytic activity of MKK7, and that this suppressive action of RASSF7 is independent of MAPKKK activation.

RASSF7 interacts with GTP-bound N-Ras via its RA domain on UV irradiation. RASSF7 is an NT-RASSF protein, meaning that its RA domain is in a different physical location than the RA domains of the classical RASSF1-6
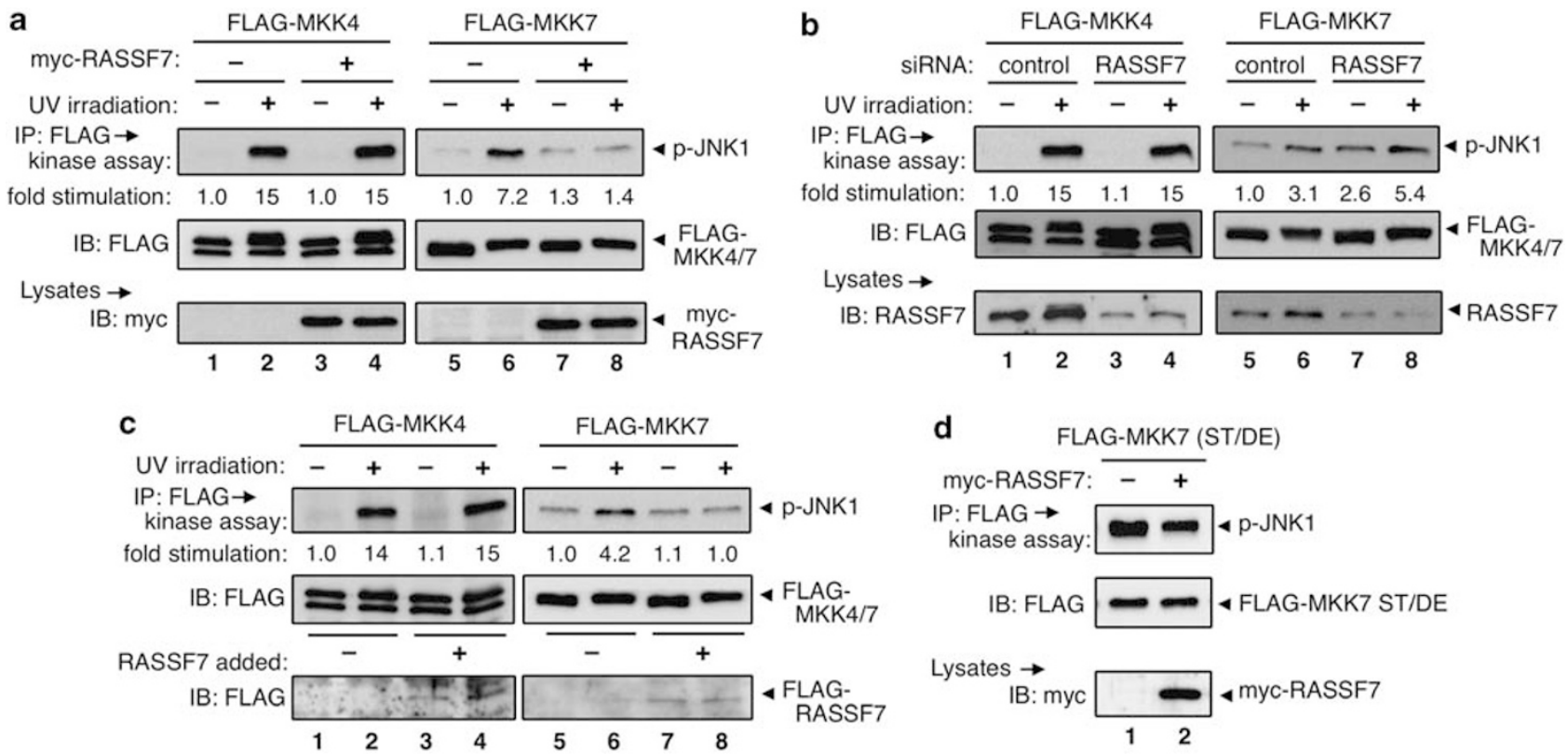

Figure 4 RASSF7 selectively inhibits UV-induced activation of MKK7. (a) RASSF7 overexpression. HeLa cells were co-transfected with FLAG-MKK4 (lanes 1-4) or FLAG-MKK7 (lanes 5-8), plus myc-mock (-) or myc-RASSF7 ( +), and cultured. The cells were exposed to UV $\left(100 \mathrm{~J} / \mathrm{m}^{2}\right)$ and cultured for $1 \mathrm{~h}$. Activated MKK4 or MKK7 were precipitated with anti-FLAG beads and subjected to an in vitro kinase assay using GST-JNK1 as a substrate. Lysates and precipitated proteins were immunoblotted to detect the indicated proteins. (b) RASSF7 siRNA. HeLa cells treated with control or RASSF7 siRNA for 24 h, co-transfected with FLAG-MKK4 (lanes 1-4) or FLAG-MKK7 (lanes 5-8) for another $24 \mathrm{~h}$. MKK activity was assessed using the in vitro kinase assay as for a. (c) Addition of RASSF7 protein. HeLa cells transfected with FLAG-MKK4 (lanes 1-4) or FLAG-MKK7 (lanes 5-8), exposed to UV $\left(100 \mathrm{~J} / \mathrm{m}^{2}\right)$, and cultured for $1 \mathrm{~h}$. To prepare control or RASSF7 protein, 293T cells were transfected with FLAG-mock or FLAG-RASSF7, and RASSF7 protein was precipitated with anti-FLAG and eluted with FLAG peptide. FLAG-MKK beads were mixed with the FLAG-mock or FLAG-RASSF7 elutants and subjected to in vitro MKK activity assays as for $\mathbf{a}$. (d) Inhibition of constitutively active MKK7. HeLa cells co-transfected with FLAG-MKK7/DE plus myc-mock or myc-RASSF7. MKK7 activity was determined as for a 
a
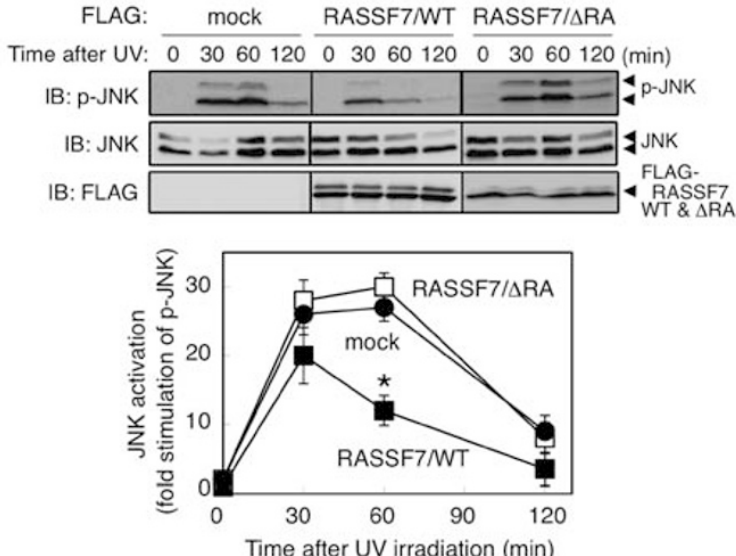

b

\begin{tabular}{l} 
myc-RASSF7 \\
\cline { 2 - 2 } FLAG-Ras: - Ha $\mathrm{Ki} \quad \mathrm{N}$ R RalA Rit Rin
\end{tabular}
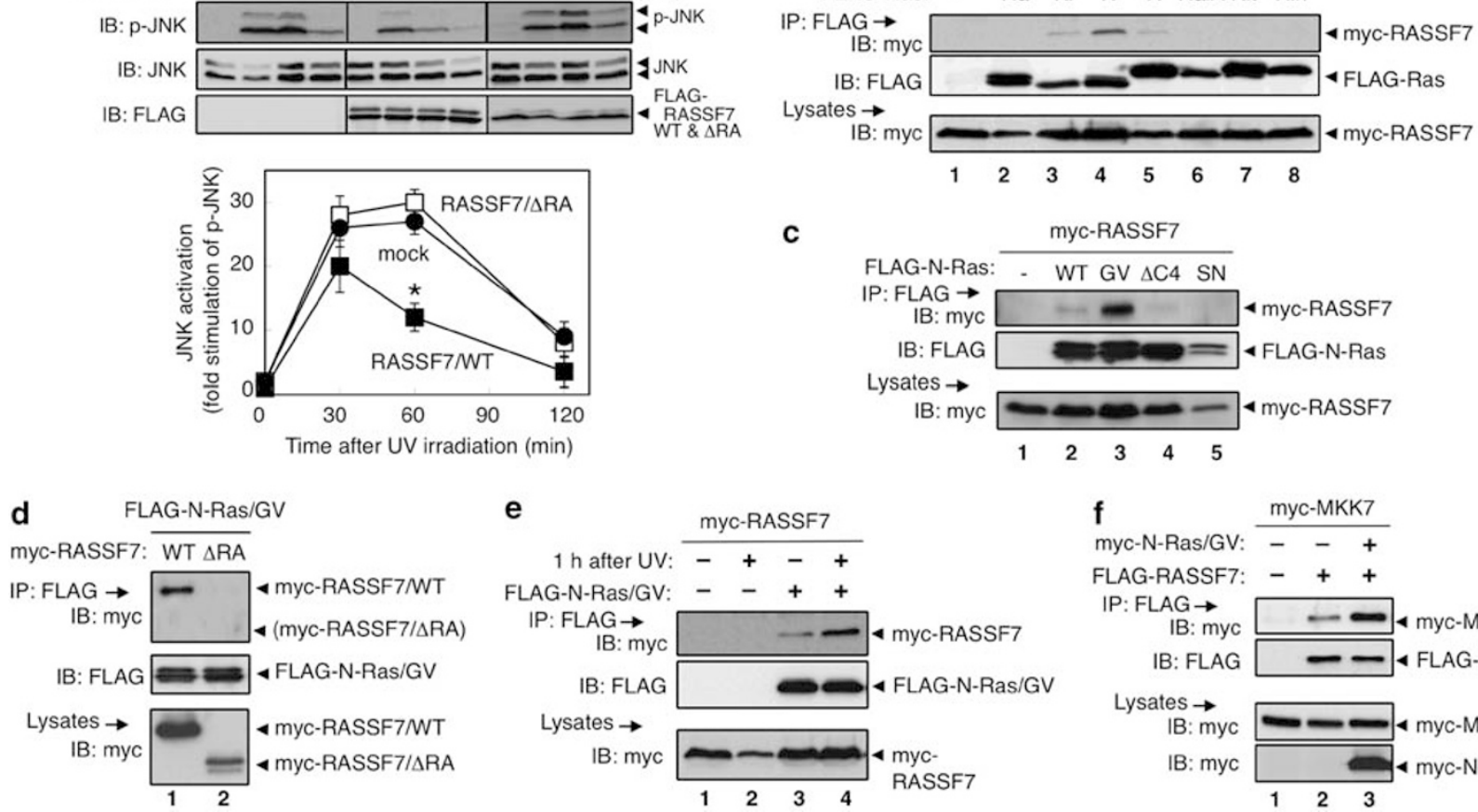

Figure 5 RASSF7 uses its RA domain to interact preferentially with GTP-bound N-Ras. (a) RASSF7/RA domain-dependent JNK activation. HeLa cells transfected with FLAG-mock, FLAG-RASSF7 (WT), or FLAG-RASSF7/ RRA were exposed to UV $\left(100 \mathrm{~J} / \mathrm{m}^{2}\right)$, and cultured for the indicated times. Lysates were immunoblotted to detect the indicated proteins. Expression of RASSF7 was confirmed using anti-FLAG Ab. JNK activation was evaluated as for Figure 2a. ${ }^{*} P<0.05$, compared with control mock transfection. (b) Specificity for N-Ras. 293T cells were co-transfected with FLAG-tagged members of the Ras family (as indicated) plus myc-RASSF7 were exposed to UV $\left(100 \mathrm{~J} / \mathrm{m}^{2}\right)$, and cultured for $1 \mathrm{~h}$. Lysates and precipitated proteins with anti-FLAG beads were immunoblotted to detect the indicated proteins. (c) Specificity for GTP-N-Ras. 293 cells were co-transfected with myc-RASSF7 plus FLAG-N-Ras, FLAG-N-Ras/G12V (constitutively active Ras), N-Ras/ $\triangle$ C4 (non-farnesylated Ras) or N-Ras/SN (constitutively inactive Ras). Cells were analyzed as for b. (d) Requirement for RASSF7 RA. 293T cells were co-transfected with myc-RASSF7 or myc-RASSF7/DRA plus FLAG-N-Ras/G12V. Cells were analyzed as for b. (e) Enhancement by UV. 293T cells were co-transfected with myc-RASSF7 plus FLAG-N-Ras/G12V, exposed to UV $\left(100 \mathrm{~J} / \mathrm{m}^{2}\right)$ and cultured for $1 \mathrm{~h}$. Cells were analyzed as for $\mathbf{b}$. (f) Enhancement of interaction between RASSF7 and MKK7 by N-Ras. 293T cells were co-transfected with myc-MKK7 plus FLAG-RASSF7 with/without myc-N-Ras/G12V, cultured for $48 \mathrm{~h}$. Cells were analyzed as for $\mathbf{b}$

proteins (Supplementary Figure S4a). Furthermore, the RA domain of RASSF7 has a unique sequence, as defined by ClustalW sequence analysis (Supplementary Figure S4b). We examined whether the RA domain of RASSF7 was responsible for its inhibitory actions. As expected from our knockdown experiments (see Figure 2a), overexpression of full-length WT RASSF7 significantly attenuated UV-induced JNK activation (Figure 5a). However, RASSF7 lacking the RA domain (RASSF7/ $\triangle R A$ ) was not able to inhibit JNK activation, indicating that the RA domain is required for RASSF7's negative regulation of JNK.

The RA domain shared by the classical RASSF proteins allows preferential interaction of these proteins with Ki-Ras. ${ }^{1,2}$ We co-transfected 293T cells with FLAG-tagged forms of various members of the Ras family, together with myc-tagged RASSF7. We then performed immunoprecipitation assays revealing that RASSF7 was capable of binding tightly to $\mathrm{N}$-Ras (Figure $5 \mathrm{~b}$, lane 4) but only weakly to Ki- or R-Ras (lanes 3 and 5). Moreover, RASSF7 appeared to interact preferentially with the GTP-bound form of N-Ras, because a constitutively active mutant (N-Ras/G12V; Figure 5c, lane 3) was able to bind to RASSF7, but the same was not true for a constitutively inactive mutant (N-Ras/S17N, lane 5) or a non-farnesylated mutant ( $\mathrm{N}-\mathrm{Ras} / \Delta \mathrm{C} 4$, lane 4$)$. As expected,
RASSF7 required the RA domain for its interaction with $\mathrm{N}-$ Ras/G12V because the RASSF7/DRA mutant failed to associate with the active $\mathrm{N}$-Ras (Figure $5 \mathrm{~d}$, lane 2). To determine whether RASSF7 interaction with N-Ras was enhanced by cellular stress, we UV-irradiated 293T cells co-transfected with FLAG-tagged N-Ras/G12V and myc-tagged RASSF7. Indeed, the interaction between RASSF7 and N-Ras/G12V was enhanced following UV irradiation (Figure $5 \mathrm{e}$, lanes 3 versus 4 ), indicating that stress stimuli drive an RA-mediated interaction between RASSF7 and $\mathrm{N}$-Ras. As shown in Figure $5 \mathrm{f}$, this interaction was enhanced in the presence of activated N-Ras, despite the unchanged protein level of RASSF7 and MKK7.

Anti-apoptotic effects of $\mathrm{N}$-Ras are mainly mediated through RASSF7. N-Ras is a GTPase that mediates survival by regulating the duration of JNK and p38 activity. ${ }^{28}$ Our data showed that RASSF7 interacted with GTP-bound N-Ras through its RA domain. We therefore investigated whether RASSF7 was involved in mediating the anti-apoptotic effects of $\mathrm{N}$-Ras. First, we determined whether $\mathrm{N}$-Ras knockdown affected stress-induced JNK activation and found that the elevation of p-JNK induced by UV was enhanced in N-Ras-knockdown cells (Figure 6a), just as it 

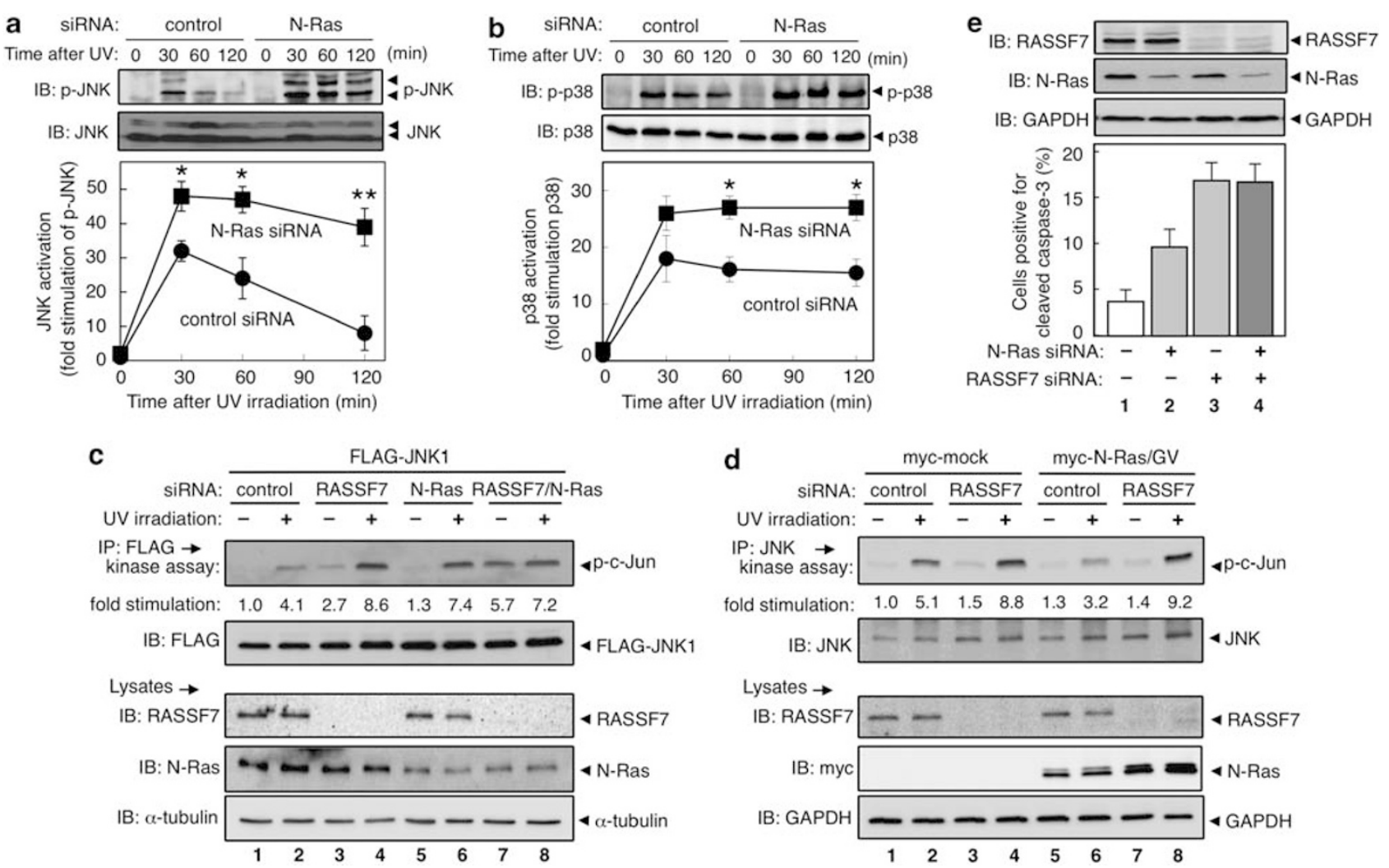

Figure 6 N-Ras and RASSF7 cooperate to inhibit UV-induced JNK activation and apoptosis. (a and $\mathbf{b}$ ) Increased p-JNK but unaltered p-p38. HeLa cells treated with control or N-Ras siRNA were exposed to UV $\left(100 \mathrm{~J} / \mathrm{m}^{2}\right)$, and cultured for the indicated times. Lysates were immunoblotted (top) to detect the total JNK and p-JNK (a), and total p38 and p-p38 (b). Results were quantitated by densitometry as for Figures $2 a$ and $b$. ${ }^{*} P<0.05$ and ${ }^{* *} P<0.01$, compared with control siRNA. (c) Increased c-Jun phosphorylation. HeLa cells treated with control or N-Ras siRNA for $24 \mathrm{~h}$ were transfected with FLAG-JNK1 for $24 \mathrm{~h}$. Cells were exposed to UV $\left(100 \mathrm{~J} / \mathrm{m}^{2}\right)$ and cultured for $1 \mathrm{~h}$. Lysates were precipitated with anti-FLAG beads, and precipitated JNK1 proteins were subjected to in vitro kinase assays as for Figure 2c. (d) Decreased c-Jun phosphorylation with N-Ras/G12V. HeLa cells treated with control or RASSF7 siRNA for $24 \mathrm{~h}$ were transfected with myc-N-Ras/G12V, cultured for $24 \mathrm{~h}$. Cells were exposed to UV $\left(100 \mathrm{~J} / \mathrm{m}^{2}\right)$ and cultured for $1 \mathrm{~h}$. Precipitated JNK1 proteins using anti-JNK sepharose were subjected to in vitro kinase assays as for Figure $2 \mathrm{c}$. (e) Caspase-3 cleavage. HeLa cells treated with N-Ras and/or RASSF7 siRNAs were exposed to UV $\left(100 \mathrm{~J} / \mathrm{m}^{2}\right)$, and cultured for $3 \mathrm{~h}$. Lysates were immunoblotted to detect the indicated proteins as for Figure $1 \mathrm{~b}$. Cells positive for cleaved caspase- 3 were quantitated as for Figures $1 \mathrm{c}$ and $d$

was in RASSF7-knockdown cells (see Figure 2a). Sustained p38 activation induced by UV was also enhanced in N-Rasknockdown cells (Figure $6 \mathrm{~b}$ ) in contrast to RASSF7knockdown cells (see Figure 2b). We further examined JNK activation in cells overexpressing JNK using the in vitro kinase assay with GST-c-Jun as the substrate. The UVinduced activation of transfected JNK (Figure 6c, lane 2) was enhanced not only by RASSF7 knockdown (lane 4) but also by N-Ras knockdown (lane 6). However, there was no synergistic enhancement in N-Ras/RASSF7 doubleknockdown cells (lane 8). Furthermore, we examined whether activated N-Ras affected UV-induced JNK activation (Figure $6 \mathrm{~d}$ ) and found that the elevation of p-c-Jun induced by UV was downregulated in cells over-expressing $\mathrm{N}$-Ras (lane 6), and this effect was totally cancelled with RASSF7 knockdown (lane 8). We also investigated the effects of RASSF7 and N-Ras knockdown on stress-induced apoptosis by measuring cleaved caspase-3. Although UV-induced apoptosis was clearly enhanced by either RASSF7 or N-Ras knockdown (Figure 6e, lanes 2 and 3), the double-knockdown cells exhibited no more than an additive effect on cell death (lane 4). These results suggest that the anti-apoptotic effect of N-Ras is mediated mainly through RASSF7. Thus, we can conclude that RASSF7 acts in concert with $\mathrm{N}$-Ras to negatively regulate UV-induced JNK activation and thus deliver an anti-apoptotic signal.

RASSF7 degradation via the ubiquitin-proteasome pathway can promote JNK activation and apoptosis. Our findings suggest that, if endogenous RASSF7 were persistently present during stress conditions, it could impede the progress of JNK-dependent apoptosis. It would thus be logical for the cell to have a mechanism in place to inactivate RASSF7 when the cell simply had to die. To determine whether such a mechanism might involve expression of the RASSF7 protein, we evaluated whether the quantity of RASFF7 protein present in HeLa cells was altered after UV irradiation. We found that RASSF7 protein levels were constant for about $1 \mathrm{~h}$ after UV exposure but decreased rapidly thereafter (Figure 7a). Titration studies showed that an effective reduction of RASSF7 in HeLa and 293T cells required 6-h exposure to $>50 \mathrm{~J} / \mathrm{m}^{2}$ UV (Figure $7 \mathrm{~b}$ ), a protocol corresponding to the exact intensity of UV 
a

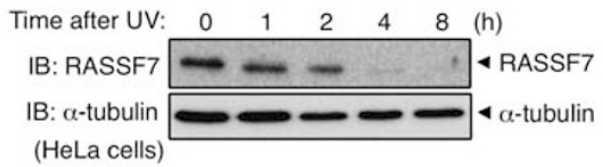

b

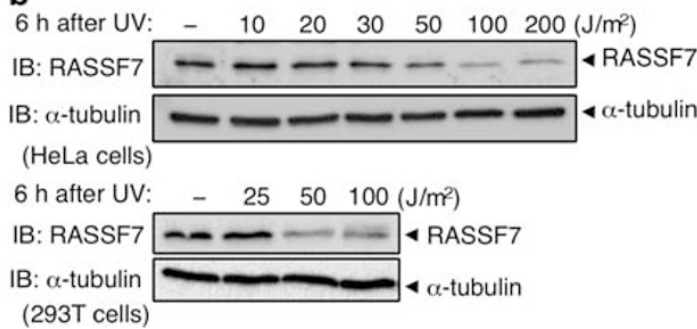

C Inhibitors: None MG132 Leupeptin

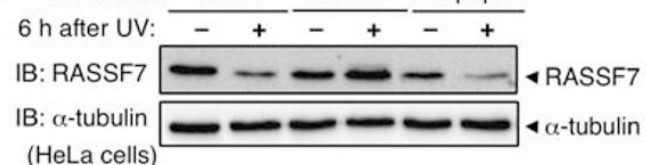

d
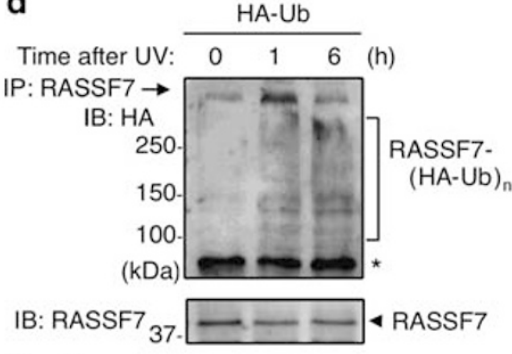

Lysates $\rightarrow$

IB: $\mathrm{HA}$

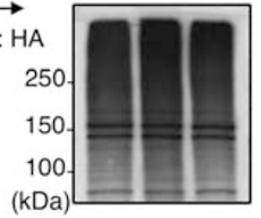

IB: RASSF7 37 A RASSF7
IB: GAPDH
(HeLa cells)

Figure 7 RASSF7 is degraded via the Ub-proteasome pathway following prolonged UV irradiation. (a and $\mathbf{b})$ Decrease in RASSF7 protein with prolonged UV irradiation. HeLa or $293 \mathrm{~T}$ cells were exposed to $100 \mathrm{~J} / \mathrm{m}^{2} \mathrm{UV}(\mathbf{a})$, or UV at the indicated intensities (b), and incubated for the indicated times (a) or $6 \mathrm{~h}$ (b). Lysates were immunoblotted to detect the indicated proteins. (c) The decrease in RASSF7 protein is proteasome-mediated. HeLa cells were exposed to UV $\left(100 \mathrm{~J} / \mathrm{m}^{2}\right)$ and cultured for $6 \mathrm{~h}$ with DMSO alone (none) or MG132 $(100 \mu \mathrm{M})$ or leupeptin $(1 \mu \mathrm{g} / \mathrm{ml})$ in $0.1 \%$ DMSO. Lysates were immunoblotted to detect the indicated proteins. (d) RASSF7 becomes polyubiquitinated. HeLa cells transfected with HA-Ub $(\mathrm{HA}-\mathrm{Ub})$ were exposed to UV $\left(100 \mathrm{~J} / \mathrm{m}^{2}\right)$ and cultured for the indicated times. Lysates and proteins immunoprecipitated with anti-RASSF7 Ab were immunoblotted to detect the indicated proteins. ${ }^{*}$ a nonspecific band

exposure needed to induce apoptosis (see Figure 1e). This UV-induced reduction in RASSF7 protein could be blocked by treatment of the cells with the proteasome inhibitor MG132, but not by treatment with the protease inhibitor leupeptin (Figure 7c), suggesting that RASSF7 is degraded via the ubiquitin (Ub)-proteasome pathway. Indeed, in response to UV irradiation, RASSF7 appeared to serve as a substrate for polyubiquitination (Figure 7d, top panel).

Next, we subjected control and RASSF7-knockdown HeLa cells to other stresses; glucose depletion, or to treatment with the DNA-alkylating agent methyl methanesulfonate or $\mathrm{H}_{2} \mathrm{O}_{2}$, and examined p-JNK levels. We found that JNK activation in response to these stress stimuli was enhanced by RASSF7 knockdown (Figure 8a), as had been observed with UV irradiation (see Figure 2a). Moreover, when we investigated the effects of these stress stimuli on levels of RASSF7 protein in HeLa cells, we noted that they induced RASSF7 degradation (Figure $8 b$ ) similar to that seen following UV irradiation (see Figure 7a).

Taken together, our results suggest that RASSF7 acts as a anti-apoptotic negative regulator of JNK activation in response to low levels of stress stimuli, but that RASSF7 protein is then degraded via the Ub-proteasome pathway as stress becomes prolonged, allowing the cell to undergo JNK-mediated apoptosis.

\section{Discussion}

In addition to upstream MAPKKKs and downstream JNK kinases, several other proteins are known to associate with MKK7, including GADD45 $\beta$ and C-FLIP ${ }_{L} .{ }^{16,17}$ These proteins inhibit MKK7 through different mechanisms: GADD45 $\beta$ blocks the catalytic activity of MKK7, whereas C-FLIP $\mathrm{L}_{\mathrm{L}}$ disrupts the interaction between MKK7 and the upstream MEKK1. Our results indicate that RASSF7 inhibits MKK7 activity via yet another unique mechanism. RASSF7 prefers to associate with the phosphorylated form of MKK7, rather than its nonphosphorylated form. Moreover, RASSF7 promotes MKK7 phosphorylation or maintenance of MKK7's phosphorylated state even in the absence of stress stimuli (see Figure 3). This RASSF7-MKK7 association inhibits subsequent JNK phosphorylation by activated MKK7 in a manner that is independent of MAPKKK activation (see Figure 4). On the basis of these findings, we have proposed a model of RASSF7's role in JNK signaling as illustrated in Figure 8c. Under resting conditions, the majority of MKK7 protein exists in its dephosphorylated form because upstream kinases are largely inactive. However, even under baseline conditions, there is likely a dynamic equilibrium between the phosphorylated and dephosphorylated forms of MKK7. We hypothesize that RASSF7 recognizes and interacts with the phosphorylated form of MKK7, imposing a rigid interaction that may decrease the ability of phospho-MKK7 to contact its substrate JNK and/or a phosphatase(s). Thus, phospho-MKK7 gradually accumulates in association with RASSF7, and this process is accelerated by stress stimuli. Consistent with this scenario, we found that UV irradiation phosphorylated MKK7, but failed to activate endogenous JNK in cells overexpressing RASSF7 (see Figure 4a). As well, MKK7 activity was enhanced in RASSF7-knockdown cells that had not been exposed to UV (see Figure 4b). We propose that this unique inhibitory mechanism mediated through RASSF7-MKK7 association 


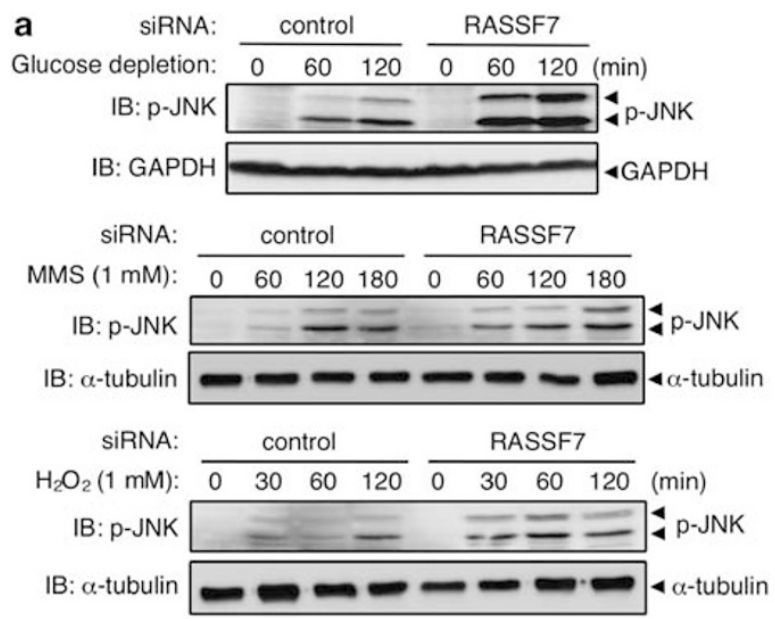

C Anti-apoptotic action of RASSF7

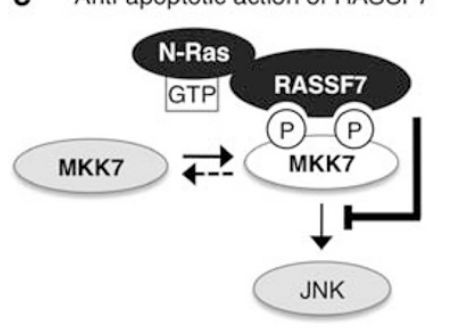

Attenuation of JNK signaling
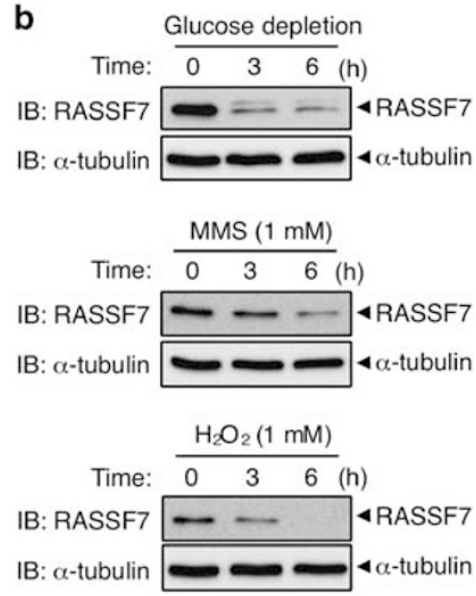

Degradation of RASSF7

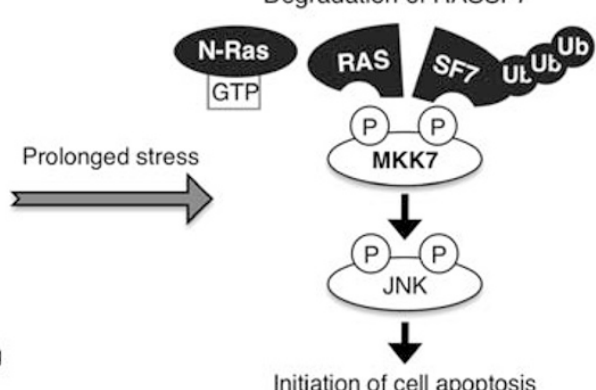

Initiation of cell apoptosis

Figure 8 RASSF7 regulates JNK activation triggered by multiple stresses, and prolonged stresses induce RASSF7 degradation. (a) RASSF7 knockdown increases JNK activation induced by non-UV stresses. HeLa cells treated with control or RASSF7 siRNA were exposed to the indicated stresses for the indicated times. Lysates were immunoblotted to detect the indicated proteins. (b) Prolongation of multiple stresses induces RASSF7 degradation. HeLa cells were exposed to the indicated stresses for the indicated times, and lysates were immunoblotted to detect the indicated proteins. (c) A model for the temporary anti-apoptotic effects of RASSF7 on JNK signaling. Schematic illustration of the relationships among the RASSF7, N-Ras, MKK7 and JNK proteins. Under resting or low stress conditions (left), the majority of MKK7 protein exists in its dephosphorylated form. RASSF7, in concert with N-Ras, prevents the phosphorylated form of MKK7 from accessing and/or activating its downstream substrate JNK. However, when stress is prolonged (right), the inhibitory action of RASSF7 on MKK7 is eliminated because of degradation of the RASSF7 protein via the Ub-proteasome pathway. In the absence of RASSF7, phosphorylated MKK7 activates JNK, triggering apoptosis

contributes anti-apoptotic regulation that helps to protect cells from inappropriate JNK activation.

Recent reports have shown that the early transient phase of JNK activation $(<1 \mathrm{~h})$ is responsible for cell survival, whereas its later and sustained phase (1-6h) mediates pro-apoptotic signaling resulting in cell death. ${ }^{29,30}$ Our data are consistent with these findings, in that JNK activation was most enhanced during the sustained phase in RASSF7-knockdown cells, and these cells were highly sensitive to cellular stress. We detected the presence of cleaved caspases, which are prototypical markers of apoptosis, as early as 3 or $6 \mathrm{~h}$ after UV irradiation (see Figures 1 and 2). We also confirmed that sustained JNK activation is required for UV-induced apoptosis in our time-course study employing SP600125 (see Figures $2 e$ and f). SP600125 in sustained phase completely suppressed UV-induced apoptosis in RASSF7-knockdown cells, implying that prolonged JNK phosphorylation is necessary for the initiation of apoptosis. Thus, our data suggest that a signal involving RASSF7 helps to protect cells against stress-induced apoptosis by attenuating the magnitude and duration of JNK (and MKK7) activity, especially during the later phase.
Although classical RASSF members bind most often to Ki-Ras, ${ }^{1,2}$ we showed that RASSF7 binds preferentially to $\mathrm{N}$-Ras. This bias may be due to differences in the amino acid sequence of the RASSF7 RA domain (see Supplementary Figures S4a and b). On the basis of our data, we suggest that RASSF7 coordinates with GTP-bound N-Ras to constitute an anti-apoptotic mechanism that promotes cell survival. In this study, we have not elucidated precisely how the interaction between RASSF7 and N-Ras is regulated under stress conditions. Generally, Ras must be in its GTP-bound form to bind to its effectors. However, we observed that the interaction of the constitutively active N-Ras/G12V mutant with RASSF7 was further enhanced by UV irradiation. It is possible that UV exposure changes the posttranslational modification or subcellular localization of RASSF7, or its interaction with a scaffold protein. It has been previously shown that posttranslational modification of the Raf is required for its binding to Ras. ${ }^{31}$ Further study will be required to elucidate how external stresses initiate the intracellular interaction of RASSF7 and N-Ras.

Persistence of this survival signaling would be a disadvantage when there was a pressing need to remove a damaged 
cell by apoptosis. Our data showed that levels of RASSF7 protein were dramatically decreased when stress became sustained (see Figures 7 and 8). This decrease in RASSF7 appeared to be due to protein degradation mediated through the Ub-proteasome pathway (see Figure 7c). Many other proteins reportedly undergo proteasomal degradation under stress conditions so that apoptosis can be induced effectively. ${ }^{32-34}$ Further study will be required to elucidate how sustained stress signaling initiates RASSF7 ubiquitination, and to determine the identity of the E3 Ub ligase involved.

\begin{abstract}
Materials and Methods
Cell culture, transfection and UV irradiation. HeLa and 293T cells were maintained in Dulbecco's modified Eagle's medium containing $10 \%$ fetal calf serum (FCS), $0.16 \%$ (w/v) $\mathrm{NaHCO}_{3}, 0.6 \mathrm{mg} / \mathrm{ml}$ L-glutamine, $100 \mu \mathrm{g} / \mathrm{ml}$ streptomycin and $100 \mathrm{IU} / \mathrm{ml}$ penicillin at $37^{\circ} \mathrm{C}$ in $95 \%$ air plus $5 \% \mathrm{CO}_{2}$. Cells were transfected with $1 \mu \mathrm{g}$ (35-mm dish) or $3 \mu \mathrm{g}$ (60-mm dish) of plasmid DNAs for $24 \mathrm{~h}$ (HeLa cells) or for $48 \mathrm{~h}$ (293T cells), or with $100 \mathrm{pmol}$ (35-mm dish) or $300 \mathrm{pmol}$ (60-mm dish) of siRNAs for $48 \mathrm{~h}$, using LipofectAMINE 2000 (Invitrogen, Carlsbad, CA, USA). Transfected cells were rinsed twice with phosphate-buffered saline (PBS) and exposed to $10-200 \mathrm{~J} / \mathrm{m}^{2}$ UV-C in a StrataLinker 1800 (Agilent-Stratagene, Santa Clara, CA, USA). In some experiments, cells were incubated in culture medium containing $0.1 \%$ DMSO (control) or inhibitors (in DMSO) for variable times before and after UV irradiation. Inhibitors used were $1 \mu \mathrm{g} / \mathrm{ml}$ leupeptin, $100 \mu \mathrm{M}$ MG132 (both from Sigma, St Louis, MO, USA), $20 \mu \mathrm{M}$ SP600125 (Enzo Life Sciences, Plymouth Meeting, PA, USA) and $20 \mu \mathrm{M}$ SB203580 (Calbiochem, Darmstadt, Germany).
\end{abstract}

Database analysis and construction of plasmids. The accession number for RASSF7 is BC106922. Alignment of the RASSF7 protein amino acid sequence to related protein sequences was carried out using ClustalW and viewed in GeneDoc. Sequences for RASSF7, Ras (Ha, Ki, N, R, RalA, Rit, Rin), JNK1, $M K K 4, M K K 7$, and Ub were isolated by polymerase chain reaction (PCR) using human whole-brain cDNA (Clontech, Mountain View, CA, USA), and cloned into pCMV-FLAG PCMV-myc, pCMV-HA or pGEX-6p-1 to generate expression vectors. pmyc-Ha-Ras/G12V was created in a previous study. ${ }^{35}$ The point-mutated forms of MKK7 (ST/DE, ST/AA, STS/AAA) and various forms of N-Ras (G12V, $\Delta C 4$ and $\mathrm{S} 17 \mathrm{~N}$ ) were generated by one day PCR as previously described. ${ }^{36}$

RNAi. HeLa cells were transfected with 25-nucleotide oligomers composed of $5^{\prime}$-UAAUUCGUGCCAGCCUCCCUGUAAA-3'/5'-UUUACAGGGAGGCUGGCA CGAAUUA-3' for RASSF7 (363-387 nt) knockdown, or 5'-CCCACCAUAGAGG AUUCUUACAGAA-3'/5'-UUCUGUAAGAAUCCUCUAUGGUGGG-3' for N-Ras (354-378 nt) knockdown. Oligomers were designed by Block-iT RNAi Designer siRNA (Invitrogen). The Stealth RNAi negative control (Invitrogen) was used as a control dsRNA.

Antibodies. Anti-JNK, anti-p38 and anti-Ub polyclonal antibodies (pAbs) were from Santa Cruz Biotechnology (Santa Cruz, CA, USA). Anti-cleaved caspase-3, anti-cleaved caspase-9, anti-cleaved PARP, anti-phospho JNK, anti-phospho p38 and anti-phospho c-Jun pAbs were from Cell Signaling Technology (Danvers, MA, USA). Anti-FLAG, anti-myc and anti- $\beta$-tubulin monoclonal antibodies (mAbs) were from Sigma; anti-GST mAb from Nacalai Tesque (Kyoto, Japan), anti-HA mAb from Roche (Indianapolis, IN, USA); anti-glyceraldehyde-3-phosphate-dehydrogenase (GAPDH) mAb from Chemicon International Inc. (Temecula, CA, USA); anti$\alpha$-tubulin mAb from Molecular Probes (Eugene, OR, USA); and anti-N-Ras mAb from Santa Cruz Biotechnology.

Generation of anti-human RASSF7 mAb. A female 6-week-old Wistar rat (CLEA Japan, Inc., Tokyo, Japan) was primed with $30 \mu \mathrm{g}$ human RASSF7 protein in Titer Max Gold and boosted twice. Splenocytes were fused with PAI mouse myeloma cells using PEG 1500 (Roche). Hybridoma supernatants were screened by indirect ELISA on PVC plates coated with $50 \mu \mathrm{l}$ of $0.5 \mu \mathrm{g} / \mathrm{ml}$ RASSF7 protein. Selected positive hybridoma lines were subcloned, grown in serum-free medium (Nihon Pharmaceutical, Tokyo, Japan) supplemented with HT (Invitrogen) and purified with Protein G Sepharose (GE Healthcare, Buckinghamshire, UK).
Immunofluorescence and microscopy. HeLa cells transiently transfected with siRNAs were cultured on a poly-L-lysine-coated coverglass (15-mm diameter) and washed three times with PBS, followed by fixation in $4 \%$ paraformaldehyde in PBS at $25^{\circ} \mathrm{C}$ for $15 \mathrm{~min}$. After permeabilization with $0.02 \%$ Triton X-100 in PBS for $10 \mathrm{~min}$, the cells were incubated with a blocking solution consisting of $2 \%$ bovine serum albumin and $2 \%$ FCS in PBS for 30 min, followed by incubation with the anti-cleaved caspase-3 pAb (Promega, Tokyo, Japan; $1 \mu \mathrm{g} / \mathrm{ml}$ diluted in blocking solution) at $37^{\circ} \mathrm{C}$ for $2 \mathrm{~h}$. The cells were washed three times with PBS and incubated for $1 \mathrm{~h}$ with rabbit Alexa-488-conjugated secondary Abs (Molecular Probes) diluted in blocking solution. After three washes in PBS, the coverglass was mounted onto a glass slide in Permafluor mounting medium (Immunon, Pittsburgh, PA, USA) and viewed on an Axio imager M1 (Carl Zeiss, Jena, Germany) using an excitation wavelength of $488 \mathrm{~nm}$. The images were merged using Adobe Photoshop (Adobe Systems, Mountain View, CA, USA). Cell nuclei were stained with 4',6-diamidino-2'-phenylindole (DAPI, Molecular Probes). To estimate numbers of cells positive for cleaved caspase-3, approximately 100-200 transfected cells per treatment were examined under a microscope by a researcher blinded to the identity of the samples.

Expression of recombinant proteins and GST pull-down assay. GST-fused forms of RASSF7, MKK7, JNK1 and c-Jun proteins were purified as described previously ${ }^{37}$ and stored in a homogenization buffer $(50 \mathrm{mM}$ Tris- $\mathrm{HCl}$ (pH 8.0), $100 \mathrm{mM} \mathrm{NaCl}, 1 \mathrm{mM}$ EDTA, $1 \mathrm{mM}$ DTT) with $0.1 \%$ Triton- $X$ or kinase buffer (see below). GST-fused RASSF7 or MKK7 was cleaved from its GST fusion partner using the Precision Protease (GE Healthcare). For GST pull-down assays, GST-fused MKK7 (or RASSF7) conjugated beads were mixed with the GST-cleaved RASSF7 (or MKK7), in the homogenization buffer with $0.1 \%$ Triton-X at $4{ }^{\circ} \mathrm{C}$ for $2 \mathrm{~h}$. The beads washed three times by the homogenization buffer were boiled and proteins bound to the beads were fractionated by SDS-PAGE.

Immunoblotting and dephosphorylation assay. HeLa and 293T cells were lysed in the homogenization buffer with $1 \%$ Triton-X, $500 \mu \mathrm{M}$ 4-(2-aminoethyl)benzenesulfonyl-flonyl-flouride (Pefabloc SC, Roche), $2 \mu \mathrm{g} / \mathrm{ml}$ aprotinin. Cell lysates were precleared with Protein G Sepharose (GE Healthcare), and incubated with anti-FLAG M2 Agarose (Sigma) or RASSF7 Ab conjugated sepharose at $4{ }^{\circ} \mathrm{C}$ for $2 \mathrm{~h}$. For preparation of RASSF7 Ab sepharose, anti-RASSF7 $\mathrm{Ab}$ was incubated with Protein $\mathrm{G}$ Sepharose overnight. The beads and precipitated proteins were washed three times in a wash buffer $(50 \mathrm{mM}$ Tris- $\mathrm{HCl}(\mathrm{pH} 8.0)$, $150 \mathrm{mM} \mathrm{NaCl}, 0.7 \%$ Triton-X, $1 \mathrm{mM}$ EDTA, $1 \mathrm{mM}$ DTT). FLAG-fused proteins and their counterparts were eluted with FLAG peptide (Sigma) in wash buffer for $30 \mathrm{~min}$. Supernatants were boiled and fractionated (along with samples of the original lysate) by SDS-PAGE. Proteins of interest were identified by immunoblotting with the appropriate Abs. For dephosphorylation assays, transfected cells were lysed in homogenization buffer fortified with $100 \mu \mathrm{M} \mathrm{Na} \mathrm{VO}_{4}$ and $50 \mathrm{mM} \mathrm{NaF}$. Immunoprecipitated myc- or FLAG-tagged RASSF7 proteins were washed and resuspended in the same buffer before the addition of $20 \mathrm{U}$ CIAP (Takara Bio Inc., Otsu, Japan). After incubation at $37^{\circ} \mathrm{C}$ for $1 \mathrm{~h}$, the reaction was terminated by adding SDS-sample buffer. Reaction mixtures were then examined by immunoblotting as described above. Detection of bands was performed using peroxidase-based chemifluorescence and charged coupled device (CCD) imager (Sony, Tokyo, Japan).

In vitro kinase activity assay. Cells were lysed in lysis buffer (20 mM Hepes $(\mathrm{pH} 7.4), 10 \mathrm{mM} \mathrm{NaCl}, 0.5 \% \mathrm{NP}-40,5 \mathrm{mM}$ EDTA, $0.05 \% \beta$-mercaptoethanol, $100 \mu \mathrm{M} \mathrm{Na}_{3} \mathrm{VO}_{4}, 50 \mathrm{mM} \mathrm{NaF}, 4 \mu \mathrm{g} / \mathrm{ml}$ aprotinin). Lysates were incubated with antiFLAG M2 Agarose (Sigma) or anti-JNK sepharose at $4{ }^{\circ} \mathrm{C}$ for $2 \mathrm{~h}$, and immunoprecipitated proteins were prepared as described above. For preparation of JNK Ab sepharose, anti-JNK Ab was incubated with Protein G Sepharose overnight. Immunoprecipitates were resuspended in kinase buffer $(50 \mathrm{mM}$ Tris- $\mathrm{HCl}$ $(\mathrm{pH} 7.4), 10 \mathrm{mM} \mathrm{MgCl}_{2}, 1 \mathrm{mM}$ EGTA) in the presence of $10 \mu \mathrm{M}$ ATP and $0.5 \mu \mathrm{g}$ GST-JNK1 or GST-C-Jun as substrates, and incubated for $20 \mathrm{~min}$ at $30^{\circ} \mathrm{C}$. The samples were subjected to immunoblotting with the appropriate antibodies as described above.

Statistics. In some experiments (Figures 1, 2, 5, 6 and Supplementary Figure 2), the statistical significance of differences between control and treated values was determined by two-tailed Student's $t$-test. 
All experiments were repeated at least three times with different batches of cell samples, and the results were fully reproducible. All data shown are representative of at least three independent trials.

\section{Conflict of interest}

The authors declare no conflict of interest.

Acknowledgements. We thank Hideko Uga for generating RASSF7 antibody. This work was supported in part by research grants from the Ministry of Education, Culture, Sports, Science and Technology (MEXT) of Japan and the Japan Society for the Promotion of Science (JSPS).

1. van der Weyden L, Adams DJ. The Ras-association domain family (RASSF) members and their role in human tumourigenesis. Biochim Biophys Acta 2007; 1776: 58-85.

2. Richter AM, Pfeifer GP, Dammann RH. The RASSF proteins in cancer; from epigenetic silencing to functional characterization. Biochim Biophys Acta 2009; 1796: 114-128.

3. Sherwood V, Recino A, Jeffries A, Ward A, Chalmers AD. The N-terminal RASSF family: a new group of Ras-association-domain-containing proteins, with emerging links to cancer formation. Biochem J 2010; 425: 303-311.

4. Mutter GL, Baak JP, Fitzgerald JT, Gray R, Neuberg D, Kust GA et al. Global expression changes of constitutive and hormonally regulated genes during endometrial neoplastic transformation. Gynecol Oncol 2001; 83: 177-185.

5. Logsdon CD, Simeone DM, Binkley C, Arumugam T, Greenson JK, Giordano TJ et al. Molecular profiling of pancreatic adenocarcinoma and chronic pancreatitis identifies multiple genes differentially regulated in pancreatic cancer. Cancer Res 2003; 63: 2649-2657.

6. Friess H, Ding J, Kleeff J, Fenkell L, Rosinski JA, Guweidhi A et al. Microarray-based identification of differentially expressed growth- and metastasis-associated genes in pancreatic cancer. Cell Mol Life Sci 2003; 60: 1180-1199.

7. Lowe AW, Olsen M, Hao Y, Lee SP, Taek Lee K, Chen X et al. Gene expression patterns in pancreatic tumors, cells and tissues. PLoS One 2007; 2: e323.

8. Tan DS, Lambros MB, Rayter S, Natrajan R, Vatcheva R, Gao $Q$ et al. PPM1D is a potentia therapeutic target in ovarian clear cell carcinomas. Clin Cancer Res 2009; 15: 2269-2280.

9. Sherwood V, Manbodh R, Sheppard C, Chalmers AD. RASSF7 is a member of a new family of RAS association domain-containing proteins and is required for completing mitosis. Mol Biol Cell 2008; 19: 1772-1782.

10. Kennedy NJ, Davis RJ. Role of JNK in tumor development. Cell Cycle 2003; 2: 199-201.

11. Weston CR, Davis RJ. The JNK signal transduction pathway. Curr Opin Cell Biol 2007; 19: 142-149.

12. Davis RJ. Signal transduction by the JNK group of MAP kinases. Cell 2000; 103: 239-252.

13. Kyriakis JM, Avruch J. Mammalian mitogen-activated protein kinase signal transduction pathways activated by stress and inflammation. Physiol Rev 2001; 81: 807-869.

14. Cavigelli M, Li WW, Lin A, Su B, Yoshioka K, Karin M. The tumor promoter arsenite stimulates AP-1 activity by inhibiting a JNK phosphatase. EMBO J 1996; 15: 6269-6279.

15. Hanada M, Kobayashi T, Ohnishi M, Ikeda S, Wang H, Katsura $\mathrm{K}$ et al. Selective suppression of stress-activated protein kinase pathway by protein phosphatase $2 \mathrm{C}$ in mammalian cells. FEBS Lett 1998; 437: 172-176.

16. Papa S, Zazzeroni F, Bubici C, Jayawardena S, Alvarez K, Matsuda S et al. Gadd45 beta mediates the NF-kappa B suppression of JNK signalling by targeting MKK7/JNKK2. Nat Cell Biol 2004; 6: 146-153.

17. Nakajima A, Komazawa-Sakon S, Takekawa M, Sasazuki T, Yeh WC, Yagita $\mathrm{H}$ et al. An antiapoptotic protein, C-FLIPL, directly binds to MKK7 and inhibits the JNK pathway. EMBO J 2006; 25: 5549-5559.
18. Kim MJ, Chae JS, Kim KJ, Hwang SG, Yoon KW, Kim EK et al. Negative regulation of SEK1 signaling by serum- and glucocorticoid-inducible protein kinase 1. EMBO J 2007; 26: 3075-3085.

19. Avruch J, Praskova M, Ortiz-Vega S, Liu M, Zhang XF. Nore1 and RASSF1 regulation of cell proliferation and of the MST1/2 kinases. Methods Enzymol 2006; 407: 290-310

20. Kitagawa D, Kajiho H, Negishi T, Ura S, Watanabe T, Wada T et al. Release of RASSF1C from the nucleus by Daxx degradation links DNA damage and SAPK/JNK activation. EMBO J 2006; 25: 3286-3297.

21. Bode AM, Dong Z. Mitogen-activated protein kinase activation in UV-induced signal transduction. Sci STKE 2003; 2003: RE2.

22. Coso OA, Chiariello M, Kalinec G, Kyriakis JM, Woodgett J, Gutkind JS. Transforming G protein-coupled receptors potently activate JNK (SAPK). Evidence for a divergence from the tyrosine kinase signaling pathway. J Biol Chem 1995; 270: 5620-5624.

23. Verheij M, Bose R, Lin XH, Yao B, Jarvis WD, Grant S et al. Requirement for ceramide-initiated SAPK/JNK signalling in stress-induced apoptosis. Nature 1996; $\mathbf{3 8 0}$ 75-79.

24. Tournier C, Whitmarsh AJ, Cavanagh J, Barrett T, Davis RJ. Mitogen-activated protein kinase kinase 7 is an activator of the c-Jun NH2-terminal kinase. Proc Natl Acad Sci USA 1997; 94: 7337-7342.

25. Ip YT, Davis RJ. Signal transduction by the c-Jun N-terminal kinase (JNK)-from inflammation to development. Curr Opin Cell Biol 1998; 10: 205-219.

26. Mansour SJ, Matten WT, Hermann AS, Candia JM, Rong S, Fukasawa K et al. Transformation of mammalian cells by constitutively active MAP kinase kinase. Science 1994; 265: 966-970.

27. Holtmann H, Winzen R, Holland P, Eickemeier S, Hoffmann E, Wallach D et al. Induction of interleukin-8 synthesis integrates effects on transcription and mRNA degradation from at least three different cytokine- or stress-activated signal transduction pathways. Mol Cell Biol 1999; 19: 6742-6753.

28. Wolfman JC, Palmby T, Der CJ, Wolfman A. Cellular N-Ras promotes cell survival by downregulation of Jun $\mathrm{N}$-terminal protein kinase and p38. Mol Cell Biol 2002; 22: $1589-1606$

29. Chang L, Kamata H, Solinas G, Luo JL, Maeda S, Venuprasad K et al. The E3 ubiquitin ligase itch couples JNK activation to TNFalpha-induced cell death by inducing c-FLIP(L) turnover. Cell 2006; 124: 601-613.

30. Ventura JJ, Hubner A, Zhang C, Flavell RA, Shokat KM, Davis RJ. Chemical genetic analysis of the time course of signal transduction by JNK. Mol Cell 2006; 21: 701-710.

31. Burgering BM, Bos JL. Regulation of Ras-mediated signalling: more than one way to skin a cat. Trends Biochem Sci 1995; 20: 18-22.

32. Bergink $\mathrm{S}$, Jaspers NG, Vermeulen W. Regulation of UV-induced DNA damage response by ubiquitylation. DNA Repair (Amst) 2007; 6: 1231-1242.

33. Harper JW, Elledge SJ. The DNA damage response: ten years after. Mol Cell 2007; 28: 739-745.

34. Hannah J, Zhou P. Regulation of DNA damage response pathways by the cullin-RING ubiquitin ligases. DNA Repair (Amst) 2009; 8: 536-543.

35. Kontani K, Tada M, Ogawa T, Okai T, Saito K, Araki Y et al. Di-Ras, a distinct subgroup of ras family GTPases with unique biochemical properties. J Biol Chem 2002; 277: 41070-41078

36. Takahashi S, Araki Y, Sakuno T, Katada T. Interaction between Ski7p and Upf1p is required for nonsense-mediated $3^{\prime}$-to- $5^{\prime}$ mRNA decay in yeast. EMBO J 2003; 22 : 3951-3959.

37. Takahashi S, Araki Y, Ohya Y, Sakuno T, Hoshino S, Kontani K et al. Upf1 potentially serves as a RING-related E3 ubiquitin ligase via its association with Upf3 in yeast. RNA 2008; 14: 1950-1958

\section{Supplementary Information accompanies the paper on Cell Death and Differentiation website (http://www.nature.com/cdd)}

